\title{
Psychometric properties of the HEXACO-PI-R self-evaluation form in Slovak translation
}

\author{
Elena Lisá ${ }^{*} \&$ Michael Dzúrik ${ }^{2}$ \\ ${ }^{1}$ Institute of Applied Psychology, Faculty of Social and Economic Sciences, Comenius University, Bratislava, Slovakia. \\ ${ }^{2}$ Faculty of Psychology, Pan-European University, Bratislava, Slovakia.
}

Received 8.09.2020; Received revised 8.06.2021; Accepted 10.06.2021;

Available online 30.06.2021

\begin{abstract}
The study aimed to verify the psychometric properties of the 100-item HEXACO-PI-R questionnaire. The sample consisted of 1624 adults aged from 16 to 79 years $(M=34.5, S D=13.35)$ who filled the paper-pen self-report form of the HEXACO-PI-R. The average internal consistency of the six factors was $\alpha=.78$ (from .72 for Openness to .81 for Honesty-Humility) and $\alpha=.60$ for facets. The Altruism scale in the Slovak translation did not reach a satisfactory internal consistency $(\alpha=.29)$. Mean values in the Slovak-speaking sample were 3.29, and standard deviations .53 for factor level and .74 at the facet level. Sex differences showed the higher Emotionality $(\mathrm{d}=.99)$ and Honesty-Humility $(\mathrm{d}=.38)$ in women. Age differences in Honesty-Humility showed a medium effect size. Factors did not inter-correlate, or they correlated weakly, except for $r=.34$ in the relationship between Agreeableness and Honesty-Humility. The factors were well distinguished from one another. The exploratory factor analysis with Promax rotation confirmed the six-factor model, which explained in total $44 \%$ of data variance, with an average loading of .60 . Individual one-factor models met most of the goodness of fit criteria in confirmatory factor analysis, but the six-factor model did not meet them. The controversy associated with assessing the internal structure of multidimensional personality inventories by confirmatory factor analysis is discussed. According to the currently published research studies, the research findings supported the reliability and internal validity of HEXACO-PI-R in Slovak translation.
\end{abstract}

Keywords: HEXACO, Honesty-Humility, personality traits, sex differences, age differences.

Address of correspondence: Elena Lisá, Institute of Applied Psychology, Faculty of Social and Economic Sciences, Comenius University, Mlynske Luhy 4, 82105 Bratislava, Slovakia. E-mail: elalisa78@gmail.com

Acknowledgment: The authors want to thank Kibeom Lee and Michael C. Ashton for their suggestions to the first manuscript draft.

\section{Introduction}

The HEXACO model writes a new chapter on personality factor models based on lexical analysis (de Vries et al., 2019). The HEXACO model of personality consists of six dimensions: Honesty-Humility (H), Emotionality (E), Extraversion (X), Agreeableness (A), Conscientiousness $(\mathrm{C})$, and Openness to Experience $(\mathrm{O})$. The measurement tool of the model is a self and an observer report questionnaire called the HEXACO Personality Inventory (Ashton et al., 2014). The HEXACO model is similar in some dimensions' characteristics with the Five-factor personality model (FFM). The X, C, and O factors differ not too much from the FFM ones. However, the $\mathrm{H}, \mathrm{A}$, and $\mathrm{E}$ differ from their FFM theory counterparts. E factor shares anxiety content with Neuroticism, but it lacks the anger-related aspects of Neuroticism, and it contains sentimentality associated with FFM Agreeableness. The A factor shares gentleness with FFM Agreeableness, and it lacks sentimentality but contains the anger-related traits from Neuroticism (Visser et al., 2016).
The $\mathrm{H}$ factor has no counterpart in the FFM. It is a unique personality trait, which correlates negatively with antisocial personality traits. Several studies empirically proved H factor validity (Aghababaei et al., 2014; Bresin \& Gordon, 2011; De Vries \& Van Kampen, 2010; Djeriouat \&Trémolière, 2014; Gaughan et al., 2012; Hodson et al., 2018; Lee et al., 2013). Factor H broadens standard views on personality traits.

In recent years, the HEXACO personality model has undergone several studies in different cultures to identify its psychometric properties and other psychosocial contexts (Lee \& Ashton, 2020; Thielmann et al., 2019). The last version of the HEXACO model questionnaires is a 100 item HEXACO-PI-R, which assesses six factors and the interstitial scale of Altruism (Lee \& Ashton, 2018). Lee \& Ashton (2018), the authors of the questionnaire, confirmed in English-speaking participants the six-factor structure of the HEXACO-100: each of the six factors was defined by its constituent facets, and each of the 25 facets was defined by its constituent items. HEXACO-PI-R factors showed low intercorrelations, with only one pair of factors $(\mathrm{H}$ and 
A) having an absolute correlation above .20 in self-report data. The factors and facets showed large self/observer convergent correlations.

Several studies brought information on the psychometric properties of the latest 100-item version of the questionnaire (Burtăverde, 2015; Záškodná \& Dostál, 2016; Bashiri et al., 2011; Barbarovič \& Šverko, 2013). Burtăverde (2015) in Romanian-speaking adults obtained a 6-factorial internal structure of HEXACO-PI-R. He also confirmed the construct validity by results of the significant relationship between the factor $\mathrm{H}$ and satisfaction with life and health; between factor $\mathrm{E}$ and job satisfaction, frustration at work and turnover; between factor $\mathrm{X}$ and job satisfaction, frustration at work, satisfaction with life and health; between factor A and job satisfaction, satisfaction with life, and frustration at work; between factor $\mathrm{C}$, job satisfaction, satisfaction with life and health; between factor $\mathrm{O}$ and satisfaction with life. Záškodná \& Dostál (2016) confirmed 6-factor structure of HEXACO-PI-R in Czech speaking students. Bashiri et al. (2011) on Iran students sample confirmed five personality factors as measured by HEXACO-PI-R (maximum likelihood analysis, Varimax rotation, and Kaiser normalization). The $\mathrm{H}$ factor failed to emerge as an independent dimension, and it was a part of the dimensions of $\mathrm{A}$ and $\mathrm{C}$. As for convergent validity, the authors confirmed significant positive correlations between the factors of HEXACO and NEO-PI (Extraversion, Conscientiousness, Agreeableness, and Openness factors of two inventories). Barbarovič \& Šverko (2013), in a sample of Croatian students, confirmed the designated six HEXACO factors, which showed expected relations with FFM traits (measured by IPIP-50). Lisá \& Kališ (2019) analyzed the psychometric properties of the Slovak translation of HEXACO-PI-R in the selection and nonselection situation (samples of employees versus candidates in the selection process). However, they concluded a need for psychometric analysis of internal structure and reliability on a larger and more representative sample of Slovak-speaking participants.

\section{Reliability}

The verification of the psychometric qualities of the HEXAO-PI-R questionnaire in the English language showed strong internal consistency values. Cronbach alfa for the six self-assessment factors ranged from .81 to .84 , and the mean value for the facets was .72 (ranging from .52 to .81) (Lee \& Ashton, 2018). The values of the internal consistency of the questionnaire factors in the Romanian translation ranged from .61 $(\mathrm{O})$ to $.87(\mathrm{X})$ (Burtăverde, $2015)$, in the Czech translation from $.76(\mathrm{O})$ to $.88(\mathrm{X})$ (Záškodná \& Dostál, 2016), in Croatian translation from .78 (E) to .85 (A) (Babarović \& Šverko, 2013). An Iranian study showed alpha values from .71 (C) to .81 (X) (Bashiri et al., 2011). Lisá \& Kališ (2019), in the Slovak translation, stated Cronbach $\alpha$ values from $.62(\mathrm{H})$ to $.73(\mathrm{E})$ in the group of job seekers, and from $.65(\mathrm{H})$ to .81 in the group of health professionals. Overall, they achieved lower Cronbach alpha values in both research files from Slovakia (Lisá \& Kališ, 2019) than in international studies (Lee \& Ashton, 2018; Babarović \& Šverko, 2013; Burtăverde, 2015). The reasons for these lower values could go with a natural field sample of working adults (not students) but also with small research samples $(\mathrm{N}=173 ; \mathrm{N}=63)$.
Validity

The construct validity of HEXACO-PI-R was demonstrated by correlation analysis of the self-assessment and others-assessment, with the results showing values of $.61(\mathrm{E}), .56(\mathrm{X}, \mathrm{O}), .46(\mathrm{H}), .52(\mathrm{C})$, and $.47(\mathrm{~A})$. Intercorrelations of the HEXACO model factors showed discriminant validity because they were generally low. $\mathrm{H}$ correlated with A in self-assessment form on the level of .30 , and no other values of correlation coefficients exceed the value of .20. Correlation coefficients in observer reports and online self-reports were slightly higher than in paper-pen self-reports (Lee \& Ashton, 2018). EFA in three samples of research participants confirmed the internal structure of the HEXACO model, with the factor loadings of the facets in all six factors above .60. The EFA in the Czech sample confirmed the existence of six factors with an achieved factor loading above .46 for all factors (Záškodná \& Dostál, 2016). The components of the HEXACO model reached satisfactory values of discriminant validity. The inter-factor correlations were relatively low (.0 to .16), except for the relationship between the factors of $\mathrm{H}$ and $\mathrm{A}$ (.28). The factors explained a total of $45.27 \%$ variance of the included variables (Záškodná \& Dostál, 2016). The EFA in the Romanian sample confirmed the internal structure of the model, explaining $60 \%$ of the variance (Burtăverde, 2015). The loadings of 24 facets ranged from .26 to .80 . Correlations between factors ranged from .01 to $.41(\mathrm{C}$ and $\mathrm{H})$. The altruism scale was not a single factor, and it divided its loadings among $\mathrm{H}, \mathrm{E}$, and $\mathrm{A}$, with loadings in the $.30 \mathrm{~s}$ and .40 s on those dimensions (Lee \& Ashton, 2018).

\section{Descriptives}

Mean values in the English-speaking sample were 3.0, and standard deviations were typically around .60 at the factor level and .80 at the facet level (Lee \& Ashton, 2018). In the Czech sample, the average value was at the level of 3.25. The average standard deviation of the factors was .56 and with facets .76 (Záškodná \& Dostál, 2016). The research in the selection and non-selection situation in Slovakia showed the average factor level of 3.44 and the average standard deviations of .42 (Lisá \& Kališ,2019).

\section{Sex and age differences}

Sex differences in English-speaking samples were found for self-reports on $\mathrm{H}$ and $\mathrm{E}$, with a higher level in women (Lee \& Ashton, 2018). In the Czech sample, women scored higher in factors $H(d=-.32), E(d=-.92)$ and men higher in factor A $(\mathrm{d}=.33)$ (Záškodná \& Dostál, 2016). Research in Romania found significant differences between men and women in factor $\mathrm{E}(\mathrm{d}=-.90)$ and Altruism ( $(d=$-.39) (Burtăverde, 2015). The Iranian sample showed statistically significant sex differences in factors $\mathrm{E}$ and $\mathrm{C}$ and the scale of Altruism (higher in women) and factor A (in favor of men) (Bashiri et al., 2011). According to the most recent findings of sex differences in HEXACO traits across countries and ethnicities (Lee \& Ashton, 2020), women averaged substantially higher than men in E $(\mathrm{d}=.84)$ and in $\mathrm{H}(\mathrm{d}=.37)$. It showed up that societal characteristics of the nations could influence the intensity of sex differences in factor E. Although factor A showed a slight sex difference $(\mathrm{d}=-.12)$, men averaged higher in some of its facets, e.g., Patience $(\mathrm{d}=-.28)$. Also, $\mathrm{O}$ facets Inquisitiveness (-.35) and Unconventionality (-.24) were higher in men, and the Aesthetic Appreciation was higher for women (.41). Women also scored higher in the interstitial scale of Altruism $(\mathrm{d}=.44)$. $\mathrm{H}$ showed an 
increasing trend with increasing age. The age trends in all personality factors were mainly quite similar for men and women (Ashton \& Lee, 2016).

\section{Current study}

The HEXACO-PI-R personality traits model is underused in Slovak research because of the missing psychometrically verified measuring tool. The current study aims to verify the psychometric properties of the HEXACO-PI-R self-report form in Slovak translation. According to results of previous studies, we assume predictable values of reliability, descriptive characteristics, gender and age differences, and internal validity of HEXACO-PI-R in the Slovak language.

\section{Method}

\section{Participants and Procedure}

Two hundred students of psychology collected data from 1624 participants from October 2019 till December 2019. They had to access the adults from their surroundings who voluntarily filled in the questionnaire in the paper-pen form. Students got the credits for the data in the personality psychology course as a reward. Michael Dzúrik, a student of psychology, transcribed paper data into the MS Excel database. Five hundred and sixteen (39.4\%) of the research group were men, $794(60.6 \%)$ were women, and 314 did not mention their sex. The mean age of the research participants was 34.5 years $(\mathrm{SD}=13,35)$, with a minimum of 16 and a maximum of 79 years (7 participants did not state their age). We divided the participants into three age groups: $16-25$ years $(\mathrm{N}=589)$, 26 - 55 years $(\mathrm{N}=888)$, and $56-79$ years $(\mathrm{N}=140)$. Participants filled the questionnaire anonymously. Participants signed the informed consent about anonymous and aggregate data analysis for the research study purposes.

\section{Instrument}

We used a paper form of a 100-item HEXACO-PI-R self-report questionnaire in Slovak translation (www.hexaco.org). The revision of translation after the data collection showed the reverse meaning of item 82 . Item number 82 was translated positively, unlike the original questionnaire (Lee \& Ashton, 2018), so we did not reverse it in the analysis. We reported an error in the translation to the authors of the HEXACO-PI-R. The Slovak translation of item 82 has been corrected and published on the hexaco.org webpage.

\section{Data analyses}

We processed the data through programs MS Excel, IBM SPSS Statistics 20, and JASP 0.14.1.0. Before verifying the HEXACO-PI-R internal factor structure, we cut the total sample of 1624 participants in two halves to perform exploratory factor analysis on the first half $(\mathrm{N}=812)$ and confirmation factor analysis on the second half $(\mathrm{N}=812)$. Firstly, we conducted exploratory factor analysis at the facet level. The facet-level factor analysis is considered a more robust test of the theoretical factor structure (Ørnfjord, 2018). When personality inventories are factor analyzed at the item level, there is a risk of overextraction of the number of latent factors, and conventional criteria for adequate factor structures are rarely met (factor loadings $>.40$, non-significant cross-loadings). Exploratory factor analysis followed by Promax rotation because the HEXACO factors usually correlate among themselves although weakly. To set the number of factors, we applied parallel analysis. Secondly, we performed a confirmatory factor analysis based on structural equation modeling to confirm the 6-factor structure of the model. We assumed the tested model with factors correlated among themselves. Several criteria of model fit were used (Hooper et al., 2008; Vassend \& Skrondal, 2011; Furnham et al., 2012; Xia \& Yang, 2019): SRMS with a value of 0.05 or lower, TLI with a value close to 0.95 or greater; CFI with a value close to 0.95 or greater, and RMSEA with a value of 0.08 or lower.

\section{Results}

Reliability, descriptive, sex, and age differences

The Cronbach alpha coefficients for the factors ranged from $.72(\mathrm{O})$ to $.81(\mathrm{H})$. Cronbach alpha for facets averaged .60 , ranging from .31 (O-Unconventionality) to .74 (H-Fairness). The Altruism dimension reached an internal consistency of .29 .

Table 1 Mean, Standard Deviation, and Cronbach Alpha of HEXACO-PI-R factors and facets in total sample/men/women, and Cohen's d for sex differences

\begin{tabular}{|c|c|c|c|c|c|c|c|}
\hline & $\begin{array}{c}\text { Total }(N=1624) \\
M(S D)\end{array}$ & $\alpha$ & Skewness & Kurtosis & $\begin{array}{c}\text { Men } \\
N=516 \\
M(S D)\end{array}$ & $\begin{array}{l}\text { Women } \\
N=794 \\
M(S D)\end{array}$ & $\begin{array}{c}\text { Sex diff. } \\
\text { Cohen's d }\end{array}$ \\
\hline \multicolumn{8}{|l|}{ Factors } \\
\hline Honesty-Humility (H) & $3.56(.59)$ & .81 & -.671 & .722 & $3.42(.61)$ & $3.64(.56)$ & -.38 \\
\hline Emotionality (E) & $3.17(.55)$ & .79 & -.167 & -.071 & $2.88(.51)$ & $3.37(.49)$ & -.99 \\
\hline Extraversion $(\mathrm{X})$ & $3.33(.53)$ & .80 & -.267 & .727 & $3.34(.55)$ & $3.32(.53)$ & .04 \\
\hline Agreeableness (A) & $2.96(.52)$ & .79 & -.264 & .263 & $2.97(.52)$ & $2.95(.51)$ & .03 \\
\hline Conscientiousness (C) & $3.51(.51)$ & .78 & -.235 & .198 & $3.48(.54)$ & $3.53(.49)$ & -.10 \\
\hline Openness $(\mathrm{O})$ & $3.24(.51)$ & .72 & -.016 & .180 & $3.26(.52)$ & $3.24(.51)$ & .04 \\
\hline \multicolumn{8}{|l|}{ Facets } \\
\hline (H) Sincerity & $3.64(.75)$ & .61 & -.593 & .473 & $3.59(.77)$ & $3.66(.74)$ & -.08 \\
\hline (H) Fairness & $3.67(.95)$ & .74 & -.637 & -.131 & $3.43(1.00)$ & $3.82(.88)$ & -.41 \\
\hline (H) Greed Avoidance & $3.41(.86)$ & .73 & -.521 & -.028 & $3.28(.85)$ & $3.48(.85)$ & -.24 \\
\hline (H) Modesty & $3.52(.68)$ & .53 & -.421 & .336 & $3.39(.73)$ & $3.61(.65)$ & -.33 \\
\hline (E) Fearfulness & $2.95(.76)$ & .59 & .013 & -.286 & $2.59(.65)$ & $3.18(.72)$ & -.85 \\
\hline (E) Anxiety & $3.31(.77)$ & .55 & -.174 & -.294 & $3.09(.79)$ & $3.47(.73)$ & -.50 \\
\hline (E) Dependence & $3.12(.82)$ & .70 & -.057 & -.412 & $2.83(.79)$ & $3.33(.78)$ & -.63 \\
\hline (E) Sentimentality & $3.31(.73)$ & .64 & -.283 & -.119 & $3.02(.73)$ & $3.52(.66)$ & -.73 \\
\hline (X) Social Self-Esteem & $3.57(.67)$ & .62 & -.572 & .655 & $3.58(.68)$ & $3.56(.68)$ & .02 \\
\hline (X) Social Boldness & $2.94(.82)$ & .72 & -.039 & -.346 & $3.07(.80)$ & $2.86(.82)$ & .25 \\
\hline
\end{tabular}




\begin{tabular}{lccccccc}
\hline & Total $(N=1624)$ & $\alpha$ & Skewness & Kurtosis & Men & Women & Sex diff. \\
\hline (X) Sociability & $3.35(.72)$ & .55 & -.262 & .003 & $3.26(.74)$ & $3.40(.71)$ & -.19 \\
(X) Liveliness & $3.46(.72)$ & .67 & -.451 & .272 & $3.46(.77)$ & $3.46(.71)$ & $.01<$ \\
(A) Forgivingness & $2.77(.72)$ & .58 & -.00 & -.299 & $2.76(.74)$ & $2.77(.72)$ & -.02 \\
(A) Gentleness & $3.18(.69)$ & .62 & -.388 & .103 & $3.10(.69)$ & $3.22(.70)$ & -.17 \\
(A) Flexibility & $2.95(.69)$ & .54 & -.216 & .102 & $2.97(.67)$ & $2.95(.70)$ & .04 \\
(A) Patience & $2.94(.74)$ & .62 & -.12 & -.118 & $3.04(.74)$ & $2.88(.70)$ & .22 \\
(C) Organization & $3.58(.77)$ & .61 & -.391 & -.268 & $3.41(.78)$ & $3.68(.74)$ & -.36 \\
(C) Diligence & $3.69(.66)$ & .61 & -.275 & -.155 & $3.72(.68)$ & $3.65(.66)$ & .10 \\
\hline (C) Perfectionism & $3.49(.71)$ & .59 & -.279 & -.111 & $3.44(.72)$ & $3.53(.71)$ & -.13 \\
(C) Prudence & $3.29(.69)$ & .60 & -.298 & .051 & $3.35(.70)$ & $3.25(.69)$ & .14 \\
(O) Aesthetic Appreciation & $3.35(.81)$ & .58 & -.192 & -.378 & $3.16(.82)$ & $3.50(.78)$ & -.43 \\
(O) Inquisitiveness & $3.29(.84)$ & .62 & -.222 & -.322 & $3.55(.80)$ & $3.13(.80)$ & .53 \\
(O) Creativity & $3.34(.77)$ & .53 & .078 & -.392 & $3.29(.74)$ & $3.37(.79)$ & -.11 \\
(O) Unconventionality & $3.00(.60)$ & .31 & .224 & .217 & $3.06(.63)$ & $2.97(.58)$ & .15 \\
Interstitial scale & & & & & & & \\
Altruism & $3.55(.58)$ & .29 & -.324 & .351 & $3.47(.57)$ & $3.64(.58)$ & -.30 \\
\hline
\end{tabular}

The average level of the factors for the whole sample was 3.29 , with an average standard deviation of .53 . The mean value of the facets was 3.29 , with a standard deviation of 74

Sex differences with medium effect size were identified for factor of $\mathrm{H}(\mathrm{d}=-.38)$, and with large effect size for factor of $\mathrm{E}(\mathrm{d}=-.99)$. Women scored higher than men in $\mathrm{H}$ and $\mathrm{E}$. As for facets of remaining factors men scored higher in $(\mathrm{X})$ Social Boldness $(\mathrm{d}=.25)$, (A) Patience $(\mathrm{d}=.22)$, and $(\mathrm{O})$ Inquisitiveness $(\mathrm{d}=.53)$. Women scored higher in (C) Organization $(\mathrm{d}=-.36),(\mathrm{O})$ Aesthetic Appreciation $(\mathrm{d}=-.43)$, and Altruism $(\mathrm{d}=-.30)$. More detailed information shows the Table 1.

Age differences showed a medium effect size in the factor of $\mathrm{H}[\mathrm{F}(2,1614)=24 ; \mathrm{p}<.001 ; \mathrm{d}=.029]$. The $\mathrm{H}$ raised with the age of participants (Table 2, Table 3). Age differences in A $[\mathrm{F}(2,1614)=10.356 ; \mathrm{p}<.001 ; \mathrm{d}=.013]$ and $\mathrm{C}$ $[F(2,1614)=5.391 ; p=.005 ; d=.007]$ were statistically significant, but with no effect size. There were no age differences in factors of $\mathrm{E}, \mathrm{X}$, and $\mathrm{O}$.

Table 2. Age differences in HEXACO factors

\begin{tabular}{lrrrccc}
\hline & \multicolumn{1}{c}{$S S$} & \multicolumn{1}{c}{$D f$} & \multicolumn{1}{c}{$M S$} & \multicolumn{1}{c}{$F$} & $p$ & $\eta^{2}$ \\
\hline $\mathrm{HH}$ & 16.172 & 2.000 & 8.086 & 24.001 & $<.001$ & .029 \\
& 543.769 & 1614.000 & .337 & & & \\
$\mathrm{Em}$ & .216 & 2.000 & .108 & .351 & .704 & .000 \\
& 496.833 & 1614.000 & .308 & & & \\
$\mathrm{Ex}$ & .769 & 2.000 & .385 & 1.374 & .253 & .002 \\
& 451.886 & 1614.000 & .280 & & & \\
$\mathrm{~A}$ & 5.499 & 2.000 & 2.749 & 10.356 & $<.001$ & .013 \\
& 428.513 & 1614.000 & .265 & & & \\
$\mathrm{C}$ & 2.769 & 2.000 & 1.385 & 5.391 & .005 & .007 \\
& 414.565 & 1614.000 & .257 & & & \\
$\mathrm{O}$ & .051 & 2.000 & .025 & .096 & .908 & .000 \\
& 427.065 & 1614.000 & .265 & & &
\end{tabular}

Notes. $\mathrm{HH}=$ Honesty-Humility; Em = Emotionality; Ex $=$ Extraversion; $\mathrm{A}=$ Agreeableness; $\mathrm{C}=$ Conscientiousness; $\mathrm{O}=$ Openess

Validity

The correlation matrix of factors showed coefficient values from -. 03 to .34 between factors $\mathrm{A}$ and $\mathrm{H}$ (Table 4). Other values of correlation coefficients did not exceed .25 . Next, we inspected the correlations of the facets (Table 5).
Table 3. Mean and Standard deviation for age groups

\begin{tabular}{clrl}
\hline Age groups & Mean & SD \\
\hline \multirow{4}{*}{ HH } & $16-25$ years & 3.44 & .637 \\
& $26-55$ years & 3.59 & .551 \\
& $56-79$ years & 3.79 & .500 \\
& Total & 3.55 & .588 \\
& $16-25$ years & 3.19 & .605 \\
Em & $26-55$ years & 3.16 & .526 \\
& $56-79$ years & 3.17 & .503 \\
& Total & 3.17 & .554 \\
& $16-25$ years & 3.33 & .609 \\
Ex & $26-55$ years & 3.34 & .479 \\
& $56-79$ years & 3.26 & .460 \\
& Total & 3.33 & .529 \\
& $16-25$ years & 2.89 & .563 \\
A & $26-55$ years & 2.98 & .486 \\
& $56-79$ years & 3.08 & .478 \\
& Total & 2.96 & .518 \\
& $16-25$ years & 3.45 & .559 \\
C & $26-55$ years & 3.54 & .477 \\
& $56-79$ years & 3.51 & .452 \\
& Total & 3.51 & .508 \\
& $16-25$ years & 3.23 & .539 \\
O & $26-55$ years & 3.24 & .508 \\
& $56-79$ years & 3.22 & .437 \\
& Total & 3.24 & .514 \\
\hline
\end{tabular}

Notes .HH $=$ Honesty-Humility; $\mathrm{Em}=$ Emotionality; $\mathrm{Ex}=$ Extraversion; $\mathrm{A}=$ Agreeableness; $\mathrm{C}=$ Conscientiousness; $\mathrm{O}=$ Openess

Table 4 Correlations among the HEXACO-PI-R Factors

\begin{tabular}{lcccccc}
\hline & $\mathbf{1}$ & $\mathbf{2}$ & $\mathbf{3}$ & $\mathbf{4}$ & $\mathbf{5}$ & $\mathbf{6}$ \\
\hline 1. Honesty-Humility & 1.00 & & & & & \\
2. Emotionality & .139 & 1.00 & & & & \\
3. Extraversion & -.033 & -.150 & 1.00 & & & \\
4. Agreeableness & .342 & -.137 & .114 & 1.00 & & \\
5. Conscientiousness & .239 & .065 & .230 & .097 & 1.00 & \\
6. Openness & .136 & -.048 & .196 & .107 & .205 & 1.00 \\
\hline
\end{tabular}

Different factors' facets inter-correlated with small practical significance, or they did not correlate at all; one correlation was medium effect sized (X4 Liveliness and C2 Diligence, $\mathrm{r}=.360$ ), and three correlations were at the border between small and medium effect size (E3 Dependence and X3 Sociability, r=.302; H4 Modesty and A2 Gentleness, $r=.301$; X1 Social Self-Esteem and C2 Diligence, $\mathrm{r}=.304$ ). 
Table 5 Correlations among the HEXACO-PI-R facets

\begin{tabular}{|c|c|c|c|c|c|c|c|c|c|c|c|c|c|c|c|c|c|c|c|c|c|c|c|c|}
\hline & 1 & 2 & 3 & 4 & 5 & 6 & 7 & 8 & 9 & 10 & 11 & 12 & 13 & 14 & 15 & 16 & 17 & 18 & 19 & 20 & 21 & 22 & 23 & 24 \\
\hline 1. $\mathrm{H} 1$ & - & & & & & & & & & & & & & & & & & & & & & & & \\
\hline 2. $\mathrm{H} 2$ & $.372 * * *$ & - & & & & & & & & & & & & & & & & & & & & & & \\
\hline 3. $\mathrm{H} 3$ & $.334 * * *$ & $.410^{* * *}$ & - & & & & & & & & & & & & & & & & & & & & & \\
\hline 4. $\mathrm{H} 4$ & $.274 * * *$ & $.324 * * *$ & $.460 * * *$ & ${ }^{*} \quad-$ & & & & & & & & & & & & & & & & & & & & \\
\hline 5. E1 & $-.091 * * *$ & .135 *** & $.057 *$ & $.118 * * *$ & - & & & & & & & & & & & & & & & & & & & \\
\hline 6. E2 & -.002 & $.083 * * *$ & .012 & $.119 * * *$ & $.374 * * *$ & ${ }^{*}-$ & & & & & & & & & & & & & & & & & & \\
\hline 7. E3 & $-.098 * * *$ & $.066^{* *}$ & -.011 & $.062 *$ & $.336 * * *$ & $.307 * * *$ & - & & & & & & & & & & & & & & & & & \\
\hline 8. E4 & $.110^{* * *}$ & $.217 * * *$ & $.143 * * *$ & $* .279 * * *$ & $.348 * * *$ & $.387 * * *$ & $.420 * * *$ & - & & & & & & & & & & & & & & & & \\
\hline 9. X1 & $.110^{* * *}$ & $.080^{* *}$ & -.010 & $-.112 * * *$ & $-.123 * * *$ & $*-275 * * *$ & -.041 & -.041 & - & & & & & & & & & & & & & & & \\
\hline 10. $\mathrm{X} 2$ & -.032 & $-.058 *$ & $-.168 * * *$ & $*-.260 * * *$ & $-.240 * * *$ & $-.267 * * *$ & .047 & $-.124 * * *$ & $.342 * * *$ & - & & & & & & & & & & & & & & \\
\hline 11. X3 & -.026 & $.041 \quad-$ & $-.063 *$ & .028 & -.037 & -.032 & $.302 * * *$ & $* .186 * * *$ & $.228 * * *$ & $.358 * * *$ & - & & & & & & & & & & & & & \\
\hline 12. $\mathrm{X} 4$ & $.125 * * *$ & $.103 * * *$ & .021 & $-.054 *$ & $-.239 * * *$ & $*-.290 * * *-$ & $-.054 *$ & -.027 & $.566 * * *$ & $.390 * * *$ & $.300 * * *$ & $*-$ & & & & & & & & & & & & \\
\hline 13. A1 & $.127 * * *$ & $.216 * * *$ & $.235 * * *$ & $* .193 * * *$ & $-.153 * * *$ & $*-184 * * *$ & $-.054 *$ & .043 & $.112 * * *$ & .008 & $.061 *$ & $.155^{* * *}$ & - & & & & & & & & & & & \\
\hline 14. A2 & $.140 * * *$ & $.139^{* * *}$ & $.190 * * *$ & $* .301 * * *$ & -.036 & -.004 & -.026 & $.176 * * *$ & .046 & $-.196 * * *$ & $.115 * * *$ & $* .091 * * *$ & $.326 * * *$ & $* \quad-$ & & & & & & & & & & \\
\hline 15. A3 & $.129 * * *$ & $.180^{* * *}$ & $.227 * * *$ & $* .246 * * *$ & -.033 & -.045 & -.037 & $.066 * *$ & $.071 * *$ & $-.180 * * *$ & .024 & $.092 * * *$ & $.298 * * *$ & $* .443 * * *$ & - & & & & & & & & & \\
\hline 16. A4 & $.165 * * *$ & .169 *** & $.167 * * *$ & $* .079 * *$ & $-.231 * * *$ & $*-288 * * *-$ & $-.182 * * *$ & $*-.106 * * *$ & $.228 * * *$ & $.052 *$ & .044 & $.296 * * *$ & $.421 * * *$ & $* .358 * * *$ & $* .404 * * *$ & - & & & & & & & & \\
\hline 17. $\mathrm{Cl}$ & $.169 * * *$ & $.220 * * *$ & $.086 * * *$ & $* .081$ ** & $.129 * * *$ & $.126^{* * *}$ & .014 & $.120 * * *$ & $.188 * * *$ & .042 & $.052 *$ & $.182 * * *$ & .012 & .021 & $.083 * * *$ & $.050 *$ & - & & & & & & & \\
\hline 18. C2 & $.185 * * *$ & $.201 * * *$ & -.024 & $-.080 * *$ & $-.123 * * *$ & * .013 & -.024 & .045 & $.304 * * *$ & $.277 * * *$ & $.163 * * *$ & $* .360$ *** & ${ }^{k} .015$ & -.042 & $-.050 *$ & $.102 * * *$ & $.322 * * *$ & - & & & & & & \\
\hline 19. $\mathrm{C} 3$ & $.136 * * *$ & $.177^{* * *}$ & $.061 *$ & .005 & $.070 * *$ & $.252 * * *$ & $.068 * *$ & $.171 * * *$ & .020 & .036 & .036 & $.090 * * *$ & ${ }^{k}-.059 *$ & -.000 & $-.080 * *$ & .010 & $.376 * * *$ & $.434 * * *$ & - & & & & & \\
\hline 20. $\mathrm{C} 4$ & $.209^{* * *}$ & $.281^{* * *}$ & $.125 * * *$ & $* .040$ & -.044 & $-.065 * *$ & $-.159 * * *$ & $*-.077 * *$ & $.154 * * *$ & .011 & $-.083 * * *$ & $* .140 * * *$ & *.120*** & $* .057 *$ & $.215^{* * *}$ & $.347 * * *$ & $.364 * * *$ & $.326 * * *$ & $.262 * * *$ & * $\quad-$ & & & & \\
\hline 21. O1 & $.169 * * *$ & $.263 * * *$ & $.203 * * *$ & $* .153 * * *$ & .018 & .079 ** & $.055 *$ & $.178^{* * *}$ & .031 & .028 & .048 & $.084 * * *$ & * $.154 * * *$ & $* .110 * * *$ & $* .135 * * *$ & ${ }^{*} .119 * * *$ & ${ }^{*} .107 * * *$ & $.142 * * *$ & $.159 * * *$ & $* .116 * * *$ & * - & & & \\
\hline 22. $\mathrm{O} 2$ & $.128 * * *$ & $.120 * * *$ & $.070 * *$ & -.012 & $-.247 * * *$ & $*-.093 * * *$ & $-.167 * * *$ & $*-.100 * * *$ & $.068 * *$ & $.099 * * *$ & .000 & $.079 * *$ & $.097 * * *$ & $* .017$ & $.091 * * *$ & *.142*** & $.060 *$ & $.205^{* * *}$ & $.101 * * *$ & $* .171 * * *$ & $* .324 * * *$ & - & & \\
\hline 23. $\mathrm{O} 3$ & $.098^{* * *}$ & $.096^{* * *}$ & .039 & -.035 & $-.058 *$ & .022 & $.062 *$ & $.091^{* * *}$ & $.134 * * *$ & $.196^{* * *}$ & $.078 * *$ & $.209 * * *$ & k .026 & -.008 & $-.070 * *$ & $.068 * *$ & -.010 & $.260 * * *$ & $.170 * * *$ & $* .028$ & $.389^{* * *}$ & $.153 * * *$ & - & \\
\hline 24. 04 & -.044 & $-.108 * * *-$ & $-.172 * * *$ & $*-.136 * * *$ & $-.158 * * *$ & *-.038 & .030 & $-.055 *$ & .047 & $.262 * * *$ & $.088 * * *$ & $* .118^{* * *}$ & ${ }^{k}-.020$ & $-.079 * *$ & $-.074 * *$ & .023 & $-.079 * *$ & $.121^{* * *}$ & $\begin{array}{ll}.039 & -\end{array}$ & -.046 & $.289 * * *$ & $229 * * *$. & $.334 * * *$ & *- \\
\hline
\end{tabular}

Notes. H1=Sincerity; H2=Fairness; H3=Greed Avoidance; H4=Modesty; E1=Fearfulness; E2=Anxiety; E3=Dependence; E4=Sentimentality; X1=Social Self-Esteem; X2=Social Boldness; X3=Sociability; $\mathrm{X} 4=$ =Liveliness; A1=Forgivingness; A2=Gentleness; A3=Flexibility; A4=Patience; C1=Organization; C2=Diligence; C3=Perfectionism; C4=Prudence; O1=Aesthetic Appreciation; O2=Inquisitiveness; O3=Creativity; O4=Unconventionality; *** $\mathrm{p}<.001 ; * * \mathrm{p}<.001 ; * \mathrm{p}<.05$ 
Exploratory factor analysis

We used exploratory factor analysis with PROMAX rotation to identify the internal component structure of 24facets HEXACO-PI-R. To determine the number of factors, we applied parallel analysis (Figure 1). Six factors explained $44.1 \%$ of the data's variance (Table 6 ).

Table 6 Factor loadings of HEXACO-PI-R facets

\begin{tabular}{|c|c|c|c|c|c|c|c|}
\hline & $X$ & E & $A$ & $H$ & C & $O$ & Uniqueness \\
\hline H: Sincerity & & & & .514 & & & .683 \\
\hline H: Fairness & & & & .609 & & & .572 \\
\hline
\end{tabular}

H: Greed

Avoidance

H: Modesty

E: Fearfulness

E: Anxiety

E: Dependence

E: Sentimentality

$\mathrm{X}$ : Social Self

.764

.466

Esteem

$\mathrm{X}$ : Social Boldness

X: Sociability

$\mathrm{X}$ : Liveliness

A: Forgiveness

A: Gentleness

A: Flexibility

A: Patience

C: Organization

$\mathrm{C}$ : Diligence

C: Perfectionism

$\mathrm{C}$ : Prudence

O: Aesthetic

Appreciation

$\mathrm{O}$ : Inquisitiveness

$\mathrm{O}$ : Creativity

$\mathrm{O}$ :

Unconventionality

Proportion Var.

Note. The applied rotation method is Promax.

All facets reached the highest loadings in the factor to which they belonged. Factor loadings ranged from .41 (O: Inquisitiveness) to .76 (H: Greed Avoidance), with a mean value of .60 .

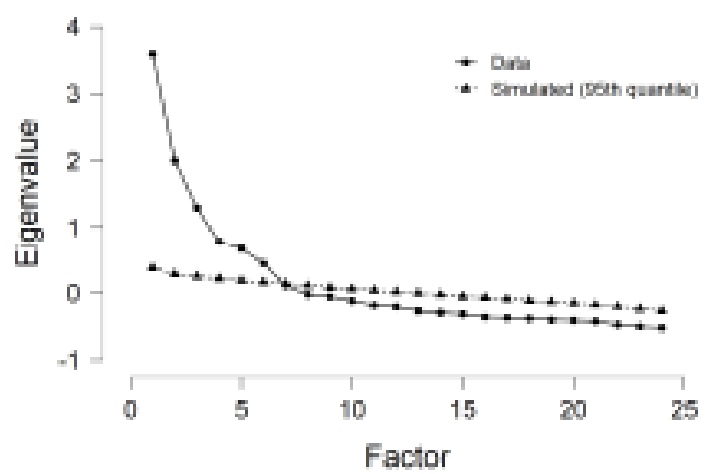

Figure 1 Scree plot

Confirmatory factor analysis

The structural parameters of CFA tested model were significant at $\mathrm{p}<.001(\mathrm{X} 2=1550.814 ; \mathrm{df}=237)$, however, the six-factor model provided poor fit to the data: $\mathrm{CFI}=$ .648 ; TLI $=.590$; RMSEA $=.083$; SRMR $=.086$. As the next step we tested the individual models separately for each factor. Fit statistics of all models estimated are summarized in Table 7. The measure of absolute data fit RMSEA was good for $\mathrm{H}$, and $\mathrm{E}$; mediocre for $\mathrm{X}$ and $\mathrm{A}$, and poor for $\mathrm{C}$ and $\mathrm{O}$. Tucker Lewis index, that is preferable for smaller samples had good value for E, and slightly less than the good fit values for other factors' models. The rest of the indexes were acceptable (CFI $>$ $.90)$ or very good $($ SRMR $<.05)$.

Table 7 Goodness of fit indices for the six HEXACO domains

\begin{tabular}{lcccccc}
\hline & $\chi^{2}$ & $p$ & $C F I$ & $T L I$ & RMSEA & SRMR \\
\hline HH & 12.7 & .002 & .949 & .846 & .081 & .035 \\
Em & 11.159 & .004 & .981 & .943 & .075 & .024 \\
Ex & 33.656 & $\mathrm{p}<.001$ & .940 & .819 & .140 & .048 \\
A & 38.047 & $\mathrm{p}<.001$ & .928 & .785 & .149 & .046 \\
$\mathrm{C}$ & 15.998 & $\mathrm{p}<.001$ & .931 & .794 & .093 & .035 \\
$\mathrm{O}$ & 19.321 & $\mathrm{p}<.001$ & .946 & .838 & .103 & .035 \\
\hline
\end{tabular}

Notes. $\mathrm{df}=2$ for all models. $\mathrm{HH}=$ Honesty-Humility; $\mathrm{Em}=$ Emotionality; $\mathrm{Ex}=$ Extraversion; $\mathrm{A}=$ Agreeableness; $\mathrm{C}=$ Conscientiousness; $\mathrm{O}=$ Openess

\section{Discussion}

The values of the internal consistency of HEXACO-PI$\mathrm{R}$ factors were slightly lower but comparable with the values measured on the Czech sample (Záškodná \& Dostál, 2016), with the smallest internal consistency for factor $O$ (Bashiri et al., 2011; Burtăverde, 2015; Lee \& Ashton, 2018; Lisá \& Kališ, 2019). The internal consistency of the six factors averaged .78 and .60 at the facet level. The highest Cronbach alpha was reached for $\mathrm{H}$, the same as in the online Canadian self-report sample (Lee \& Ashton, 2018). Usually, factor $X$ reaches the highest value of internal consistency (Bashiri et.al, 2011; Burtăverde, 2015; Lee \& Ashton, 2018; Lisá \& Kališ, 2019; Záškodná \& Dostál, 2016). The different wording of item 82 could explain lower internal consistency of $\mathrm{X}$ in the research sample compared with international studies. Because of the lower internal consistency values in some facets, we recommend for using the HEXACO-PI-R in Slovak translation to interpret the six factors and consider selected facets. The results confirmed that the Altruism dimension in the Slovak translation did not show an acceptable internal consistency value (Lisá \& Kališ, 2019). However, the internal consistency values of the six factors enable the use of the tool in the Slovak version for further research.

The average value of the factors and facets level as well as the standard deviations were comparable to the Czech language sample (Záškodná \& Dostál, 2016), which is the closest to the Slovak language. Sex differences, at both factor and facet levels, are broadly consistent with previous findings (Lee \& Ashton, 2020).

Women scored higher in $\mathrm{H}$ and $\mathrm{E}$ factors. Differences in factor $\mathrm{E}$ facets are close to those found in Romania (Burtăverde, 2015) or Canada (Lee \& Ashton, 2018). Differences in facets of the $\mathrm{H}$ factor are similar to those in results of Lee \& Ashton (2018). We found a higher score in men with a medium effect size in the facets of (A) Patience and (O) Inquisitiveness (Lee \& Ashton, 2020; Záškodná \& Dostál, 2016); and a higher score in women with a medium effect size in the facet of $(\mathrm{O})$ Aesthetic Appreciation and Altruism (Lee \& Ashton, 2020; Záškodná \& Dostál, 2016). In the mentioned studies, in contrast to the Slovak sample, there was no difference between men and women in the facet (X) Social Boldness $(\mathrm{d}=.25)$. 
Age differences confirmed the previous findings (Ashton \& Lee, 2016) when the only factor of $\mathrm{H}$ showed an increase with age (medium effect size).

Within discriminant validity, consistent with the results of other studies, we confirmed a partial overlap of the factors of $\mathrm{H}$ and A (Lee \& Ashton, 2018; Záškodná \& Dostál, 2016). Correlations between .2 and .25 also appeared between factors of $\mathrm{H}$ and C (Burtăverde, 2015; Solár \& Paulovičová, 2016) and between factors of C and X (Burtăverde, 2015). Overall, we can say that the factors of the HEXACO-PI-R are well distinguished from one another. The PCA confirmed the internal structure of the HEXACO-PI-R questionnaire in the Slovak translation. All facets saturated their factor primarily, with factor loadings above .53. Two facets also saturated the other factors, similar to the English-speaking sample (Lee \& Ashton, 2018).

Separated one-factor models met most of the goodness of fit criteria in CFA, but the whole six-factor model did not meet them, similar to Vassend \& Scrondall (2011). This findings are in line with prior research on omnibus personality inventories and recent HEXACO CFA results (Thielmann et al., 2019). Generally, there is a controversy associated with assessing the internal structure of omnibus personality inventories with CFA (Gignac, 2009; Hopwood \& Donnellan, 2010). According to Furnham et al. (2012), it is unlikely that the goodness of fit indices standards can be achieved in CFA for such complex personality models. The six-factor model of HEXACO-PI-R, similarly to the fivefactor model of NEO-PI-R (Aluja et al., 2005), shows a relatively complicated structure for the CFA conditions. Future research could explore alternatives to one-factor models on that facets and factors of a complex personality model are based, such as, e.g., HEXACO-PI-R or NEO-PIR (Vassend \& Scrondall, 2011).

\section{Limits and further recommendations}

Item 82, "I tend to feel quite self-conscious when speaking in front of a group of people. "had a different meaning in the Slovak translation: "I tend to feel quite selfconfident when speaking in front of a group of people. "Because of that, we did not reverse the item according to the scoring key. In future research on the HEXACO model in Slovak translation, we recommend working with a corrected version, available at hexaco.org website.

Another limit of the study is that it dealt only with the self-assessment form. For a complete verification of the psychometric properties of the tool, it would be appropriate to compare the results between self and other assessment forms.

The study results showed the reliability and solid internal structure of the HEXACO-PI-R questionnaire, which has no alternative in the Slovak language. For this reason, we recommend the self-report HEXACO-PI-R measuring tool in Slovak translation for further research. The use of HEXACO personality questionnaires in Slovak research is rare. Solár \& Paulovičová (2016) intended to verify the relationship of the HEXACO model (60-item) with the FFM. Still, for several reasons, they could not confirm the research hypotheses. Lisá \& Kališ (2019), when verifying the psychometric properties of the Slovak translation of the HEXACO-PI-R questionnaire in a selection and non-selection situation, concluded that the questionnaire in the Slovak translation should be verified first on a more representative sample of participants. Therefore, the current study results may help develop research about the HEXACO model in Slovak translation.

\section{Conclusion}

The verification of the psychometric properties of the 100-item HEXACO-PI-R questionnaire showed their sound quality. Internal consistency at the factor level was appropriate. The factor structure and factor intercorrelation were consistent with previous findings (Lee \& Ashton, 2018). The correlations between the six dimensions of the model were relatively weak. Sex differences confirmed the results of the recent international comparative study (Lee \& Ashton, 2020). The $\mathrm{H}$ factor showed changes in age groups as it increased with age (Ashton \& Lee, 2016). The Altruism scale in the Slovak translation did not reach a satisfactory value of internal consistency. We conclude that the Altruism scale has a slightly different meaning in the Slovak translation than in the English-speaking countries. The 100-item HEXACO-PI-R questionnaire in Slovak translation is suitable for a variety of research uses. The psychometric properties of HEXACO questionnaires alternatives (60 and 200 item versions) still need to be verified. The research findings helped support the HEXACO-PI-R structure/properties and confirmed the intercultural validity of the tool. The research findings showed that HEXACO-PI-R factors in Slovak translation could be interpreted in much the same way as in other language versions.

\section{Compliance with Ethical Standards}

The authors have no funding to disclose.

All procedures performed in studies involving human participants followed the ethical standards aligned with the 1964 Helsinki Declaration and its later amendments or comparable ethical standards.

The authors declare that they have no conflict of interest. Informed consent was obtained from all individual adult participants included in the study. All human participants were informed about the study's purpose. They filled the questionnaire in paper-pen form voluntarily and anonymously. They were informed about anonymous and aggregate data analysis for the research study purposes and expressed their agreement by signing the informed consent.

\section{References}

Aghababaei, N., Mohammadtabar, S., \& Saffarinia, M. (2014). Dirty Dozen vs. the $\mathrm{H}$ factor: Comparison of the Dark Triad and Honesty-Humility in prosociality, religiosity, and happiness. Personality and Individual Differences, 67, 6-10. http://doi.org/10.1016/j.paid.2014.03.026

Aluja, A., García, O., García, L.F., \& Seisdedos, N. (2005). Invariance of the "NEO-PI-R" factor structure across exploratory and confirmatory factor analyses. Personality and Individual Differences, 38, 1879-1889. https://doi.org/10.1016/j.paid.2004.11.014

Ashton, M., Lee, K., \& de Vries, R. (2014). The HEXACO Honesty-Humility, Agreeableness, and Emotionality Factors: A Review of Research and Theory. Personality and Social Psychology Review, 18, 139 -152. http://doi.org/10.1177/1088868314523838

Ashton, M.C. \& Lee, K. (2016). Age trends in HEXACO-PI-R self-reports. Journal of Research in Personality, 64, 102111. http://doi.org/10.1016/j.jrp.2016.08.008

Babarović, T. \& Šverko, I. (2013). The Hexaco Personality Domains in The Croatian Sample. Journal for General Social Issues, $\quad 22, \quad 397-411$. http://doi.org/10.5559/di.22.3.01

Bashiri, H., Barahmand, U., Akabri, Z.S., Ghamari, G.H., \& Vusugi, A., (2011). A Study of The Psychometric Properties and The Standardization of HEXACO 
Personality Inventory. Procedia - Social and Behavioral 30 , 1173-1176. http://doi.org/10.1016/j.sbspro.2011.10.228

Bresin, K. \& Gordon, K.H. (2011). Characterizing pathological narcissism in terms of the HEXACO model of personality. Journal of Psychopathology and Behavioral Assessment, 33, 228-235. http://doi.org/10.1007/s10862-010-9210-9

Burtăverde, V. (2015) Psychometric Properties of The Hexaco Pi-R on a Romanian Sample. The Relationship with Work Outcomes and General Outcomes. Romanian Journal of Experimental Applied Psychology, 61, 31-58. http://www.rjeap.ro/files/vol6no1/4 vol 6 i 1.pdf

de Vries, R.E. \& Van Kampen, D. (2010). The HEXACO and 5DPT models of personality: A comparison and their relationships with psychopathy, egoism, pretentiousness, immorality, and Machiavellianism. Journal of Personality Disorders, 24, 244-257. http://doi.org/10.1521/pedi.2010.24.2.244

de Vries, R.E., Ashton, M.C., \& Lee, K. (2019). New Developments in HEXACO Personality Research. Zeitschrift für Psychologie, 227, 163-165. http://doi.org/10.1027/2151-2604/a000374

Djeriouat, H. \& Trémolière, B. (2014). The Dark Triad of personality and utilitarian moral judgment: The mediating role of Honesty/Humility and Harm/Care. Personality and Individual Differences, 67, 11-16. http://doi.org/10.1016/j.paid.2013.12.026

Furnham, A., Guenole, N., Levine, S.Z., \& ChamorroPremuzic, T. (2012). The NEO Personality InventoryRevised: Factor Structure and Gender Invariance From Exploratory Structural Equation Modeling Analyses in a High-Stakes Setting. Assessment, 20, 14-23. https://doi.org/10.1177/1073191112448213

Gaughan, E.T., Miller, J.D., \& Lynam, D.R. (2012). Examining the utility of general models of personality in the study of psychopathy: A comparison of the HEXACOPI-R and NEO PI-R. Journal of Personality Disorders, 26, 513-523. http://doi.org/10.1521/pedi.2012.26.4.513

Gignac, G.E. (2009). Partial confirmatory factor analysis: Described and illustrated on the NEO-PI-R. Journal of Personality Assessment, 91, 40-47. https://doi.org/10.1080/00223890802484126

Hodson, G., Book, A., Visser, B.A., Volk, A.A., Ashton, M.C., \& Lee, K. (2018). Is the dark triad common factor distinct from low honesty-humility? Journal of Research in Personality, $73, \quad 123-129$. http://doi.org/10.1016/j.jrp.2017.11.012

Hooper, D., Coughlan, J., \& Mullen, M. (2008). Structural equation modeling: Guidelines for determining model fit. Electronic Journal of Business Research Methods, 6, 5360.

Hopwood, C.J., \& Donnellan, M.B. (2010). How should the internal structure of personality inventories be evaluated?
Personality and Social Psychology Review, 14, 332-346. doi: $10.1177 / 1088868310361240$

Lee, K. \& Ashton, M.C. (2020). Sex differences in HEXACO personality characteristics across countries and ethnicities. Journal of Personality,. http://doi.org/10.1111/jopy.12551

Lee, K., \& Ashton, M.C. (2018). Psychometric Properties of the HEXACO-100. Assessment, 25, 543-556. https://doi.org/10.1177/1073191116659134

Lee, K., Ashton, M.C., Wiltshire, J., Bourdage, J.S., Visser, B.A., \& Gallucci, A. (2013). Sex, power, and money: Prediction from the Dark Triad and Honesty-Humility. European Journal of Personality, 27, 169-184. http://doi.org/10.1002/per. 1860

Lisá, E. \& Kališ, M. (2019). The psychometric characteristics of the Hexaco-100 questionnaire used in the selection and non-selection situation. Work and Organizational Psychology 2019, Brno: Masarykova Univerzita, 144-153. http://doi.org/10.5817/CZ.MUNI.P210-9488-2019-15

Ørnfjord. M. (2018). The Norwegian HEXACO-PI-R: Psychometric properties and relationships with the Big Five Inventory. Scandinavian Psychologist, 5, e15. https://doi.org/10.15714/scandpsychol.5.e15

Sollár, T. \& Paulovičová, R. (2016). Vztáh čestnosti $k$ osobnostným faktorom Pätfaktorového modelu. Psychologia Práce a Organizácie. Nitra: Univerzita Konštantína Filozofa v Nitre.

Thielmann, I., Akrami, N., Babarović, T., Belloch, A., Bergh, R., Chirumbolo, A., Čolović, P. et al. (2019). The HEXACO-100 Across 16 Languages: A Large-Scale Test of Measurement Invariance. Journal of Personality Assessment, 1532-7752, $\quad$ https:// doi.org/10.1080/00223891.2019.1614011

Vassend, O. \& Skrondal, A. (2011). The NEO personality inventory revised (NEO-PI-R): Exploring the measurement structure and variants of the five-factor model. Personality and Individual Differences, 50, 13001304. https://doi.org/10.1016/j.paid.2011.03.002

Visser, B.A., Book, A.S., \& Volk, A.A. (2016). Is Hillary dishonest and Donald narcissistic? A HEXACO analysis of the presidential candidates' public personas. Personality and Individual Differences, 106, 281-286. http://doi.org/10.1016/j.paid.2016.10.053

Záškodná T. \& Dostál D. (2016). Sestifaktorový model osobnosti a psychometrické vlastnosti revidovaného osobnostního inventáře HEXACO, Psychologie a jeji kontexty, 7, 31-42.

Xia, Y., Yang, Y. (2019). RMSEA, CFI, and TLI in structural equation modeling with ordered categorical data: The story they tell depends on the estimation methods. Behavior Research Methods, 51, 409-428. https://doi.org/10.3758/s13428-018-1055-2 